\title{
FOXP1 and TP63 involvement in the progression of myelodysplastic syndrome with $5 q-$ and additional cytogenetic abnormalities
}

\author{
Alberto L'Abbate ${ }^{1}$, Crocifissa Lo Cunsolo ${ }^{2}$, Ettore Macrì ${ }^{2}$, Paolo luzzolino ${ }^{2}$, Cristina Mecucci ${ }^{3}$, Claudio Doglioni ${ }^{4}$, \\ Michelina Coco ${ }^{5}$, Lucia Anna Muscarella ${ }^{5}$, Simona Salati ${ }^{6}$, Enrico Tagliafico ${ }^{6}$, Carla Minoia ${ }^{7}$, Giacoma De Tullio $^{7}$, \\ Attilio Guarini ${ }^{7}$, Nicoletta Testoni ${ }^{8}$, Claudio Agostinelli ${ }^{8}$ and Clelia Tiziana Storlazzi ${ }^{1 *}$
}

\begin{abstract}
Background: The progression of low-risk del( $5 q)$ myelodysplastic syndrome to acute myeloid leukemia is increased when associated with mutations of TP53, or with additional chromosomal abnormalities. However, to date the prognostic impact and molecular consequences of these rearrangements were poorly investigated. Single additional alterations to del(5q) by balanced chromosome rearrangements were rarely found in myelodysplasia. In particular, balanced alterations involving TP63 and FOXP1 genes were never reported in the literature.

Case presentation: Here we report on a 79-year woman with an aggressive form of myelodysplastic syndrome with del (5q), no TP53 mutation, and a novel complex rearrangement of chromosome 3 in bone marrow cells. Our results revealed that the FOXP1 and TP63 genes were both relocated along chromosome 3. Strikingly, immunohistochemistry analysis showed altered protein levels, disclosing that this rearrangement triggered the expression of FOXP1 and TP63 genes. FOXP1 was also found activated in other patients with myelodysplasia and acute myeloid leukemia, showing that it is an important, recurrent event.

Conclusions: We document an apparent role of FOXP1 and TP63, up to now poorly documented, in the progression of MDS in our patient who is lacking mutations in the TP53 tumor suppressor gene normally associated with poor outcome in myelodysplastic syndrome with $5 \mathrm{q}$-. Finally, our results may suggest a possible broader role of FOXP1 in the pathogenesis and progression of myelodysplasia and acute myeloid leukemia.
\end{abstract}

Keywords: Double inversion, Myeloid leukemia, TP53, Gene activation, Chromosome 3

\section{Background}

In MDS, del(5q) was associated with a low-risk of leukemic evolution, unless it was accompanied by a mutation of TP53 [1], or by a complex karyotype (presence of $\geq 1$ additional chromosomal abnormalities) [2,3]. The most frequent single extra abnormalities to $\operatorname{del}(5 \mathrm{q})$ were: del (12p), trisomy 21 , trisomy $8, \operatorname{del}(20 q)$ [2], and the recently identified del(15)(q26.1), and $\operatorname{del}(3)(\mathrm{q} 26.1)$, respectively deleting the CHD2, and THPO genes [3]. Conversely, balanced chromosome rearrangements were rarely found as a single additional alteration to del (5q). Notably, the

\footnotetext{
* Correspondence: cleliatiziana.storlazzi@uniba.it

'Department of Biology, University of Bari, Via G.Amendola 165/A, Bari 70126, Italy

Full list of author information is available at the end of the article
}

molecular consequences and prognostic impact of each single aberration, mostly if balanced, were poorly investigated to date [4].

Here we describe a patient with an aggressive form of MDS. The BM karyotype, in addition to $5 \mathrm{q}$ deletion, showed an acquired abnormal chromosome 3 (Figure 1a). This abnormality led to the concurrent alteration of the FOXP1 and TP63 genes, never before reported in the literature.

\section{Case presentation}

The patient, a 79-year woman, was admitted to our hospital in January 2010. Her blood count showed: WBC $10 \times 10^{9} / 1$ with $1 \%$ myeloblasts, $\mathrm{Hb} 8.5 \mathrm{~g}$ per $100 \mathrm{ml}$, and platelets $135 \times 109 / 1$. Histological sections indicated that the BM was 


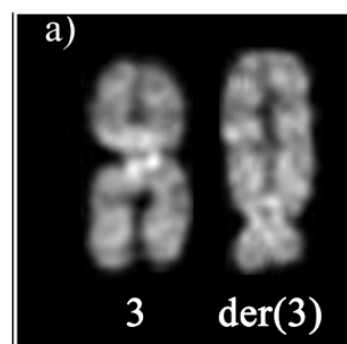

b)

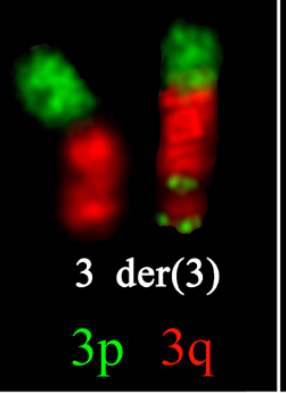

c)

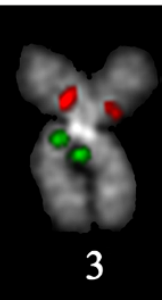

RP11-910P10 (chr3:71,183,208-71,361,561)

RP11-90I13 (chr3:106,875,308-107,035,570)

d)

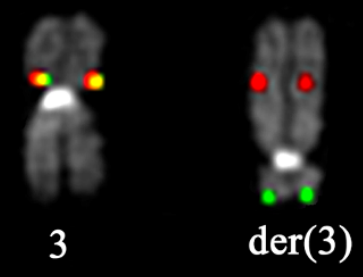

G248P80064E6 (chr3:71,230,053-71,270,407) G248P8385G8 (chr3:71,264,028-71,305,819)

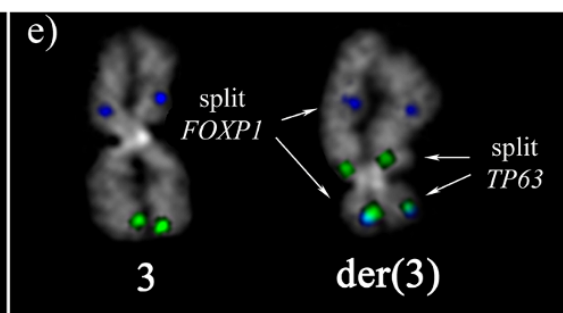

RP11-910P10 (chr3:71,183,208,71,361,561) RP11-1148C5 (chr3:189,419,877-189,573,591)

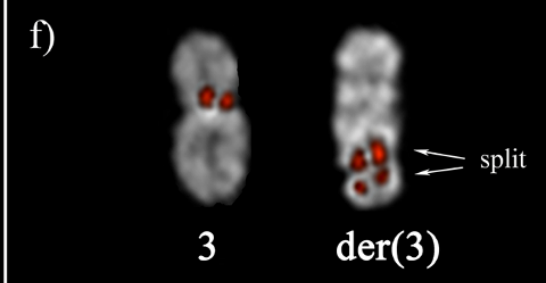

RP11-453G17 (chr3:82, 126,332-82,306,564)
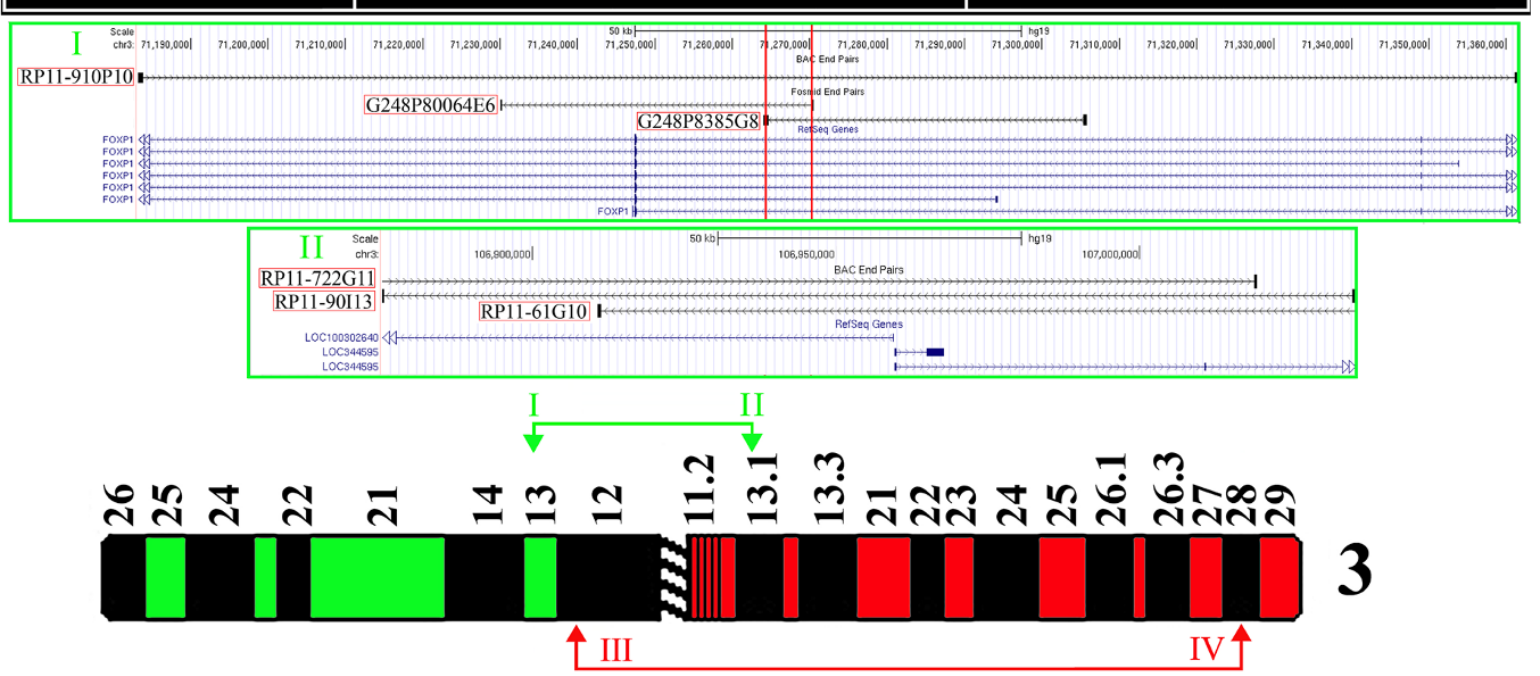

g)
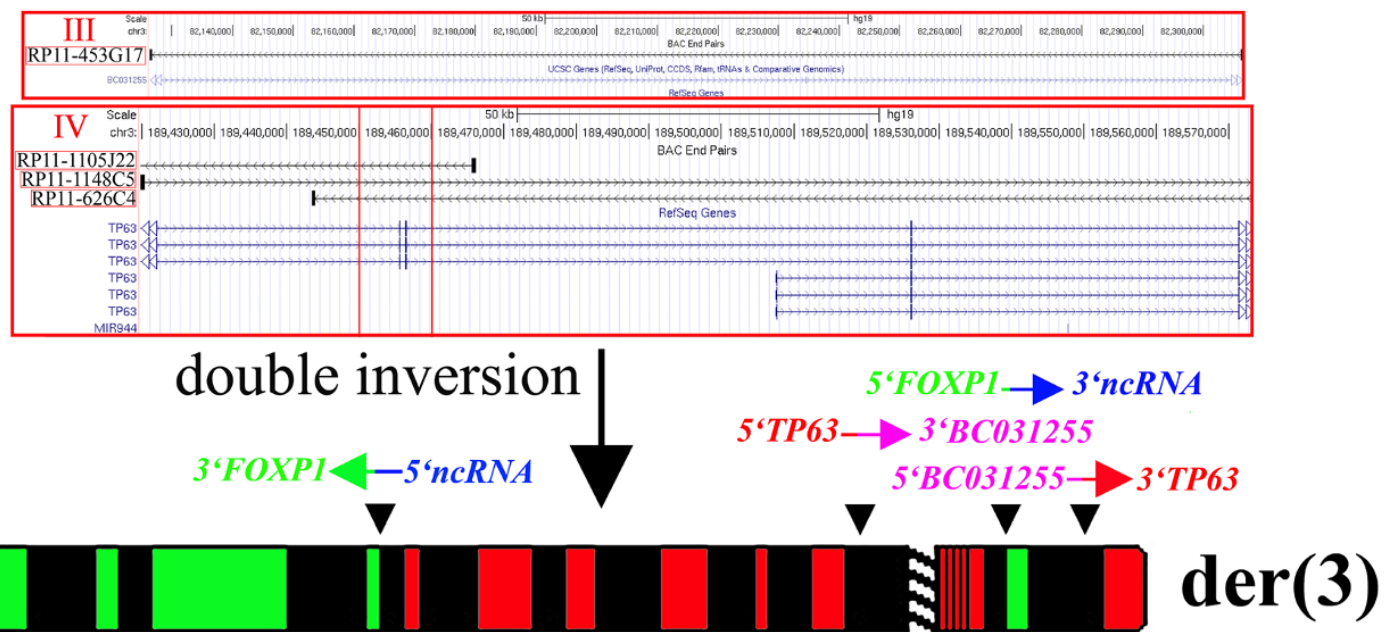

Figure 1 (See legend on next page.) 

performed as previously described [5], using commercial PCP probes specific for 3p (Kreatech, prod. No. KBI-30104, green), and 3q (Kreatech, prod. No. KBI-30105, red); (c, d) FISH experiments with BAC (c) and fosmid (d) clones to define the breakpoint regions in chromosome bands 3 p13 and 3q13.12; (e, f) FISH cohybridization experiments with probes defining the breakpoints in bands 3p12.2 (e) and 3q28 (f); (g) Schematic representation of the double inversion leading to the formation of the der(3) chromosome. I, II, III, and IV refer to contig maps of BAC and fosmid clones of the breakpoint regions in chromosome bands 3p13, 3q13.12, 3p12.2, and 3q28, respectively. The clones used in FISH are indicated by red rectangles; the red arrows point on the intervals (defined by the red vertical lines) containing the breakpoints.

cellular at $80 \%$, including numerous dystrophic megakaryocytes, reduction of normoblastic erythroid elements, and increase of myeloid immature cells with dysplastic morphology. Moreover, a diffuse and severe reticulin fibrosis was observed, and a diagnosis of myelodysplasia with myelofibrosis was made. The BM aspirate at MDS diagnosis had the karyotype: 46,XX,inv(3)(p?26q?13),del(5)(q31q35)[20]. ish del(5)(q31.2q31.2)(EGR1-). The complex chromosome 3 rearrangement was found in all the metaphases, strongly suggesting its role as a driver mutation. Notably, this patient showed neither deletions nor point mutation at TP53 (exons 4-8), known to be associated with an increased risk of leukemic evolution in MDS with del(5q) [1]. However, the patient, treated with hydroxyurea, progressed quickly towards AML in December 2011, with the following blood count: WBC $77 \times 10^{9} / 1$ with $21 \%$ myeloblasts, $\mathrm{Hb} 8.1$ g per $100 \mathrm{ml}$, and platelets 19x109/l. The karyotype at this stage was not available, due to the lack of metaphases in the BM. Immediately after, the patient died for a fatal cerebral hemorrhage.

$\mathrm{CN}$ analysis on the BM genomic DNA of the patient was accomplished on a Genome-wide human SNP array 6.0 according to manufacturer protocols (Affymetrix, Santa Clara, CA, USA). The resulting data, analyzed by the Genotyping console software V.4.1.3.840, and by Chromosome Analysis Suite V. CytoB-N1.2.0.225 using the GRCh37/hg19 genome sequence, confirmed the occurrence of a 5q23.1-q33.3 deletion. Additionally, $\mathrm{CN}$ variations within $J A K 2$ and $K M T 2 A$ (also known as $M L L)(\mathrm{CN}$ state 1 and 3, respectively) were observed. FISH, performed as previously described [5], excluded the involvement of $J A K 2$ and $K M T 2 A$ in further rearrangements leading, for instance, to fusion genes (data not shown), but it could not confirm the $\mathrm{CN}$ variation (18.8 and $32.6 \mathrm{~Kb}$, respectively). We conclude that, apart from the deletion of chromosome 5 , the complex rearrangement of chromosome 3 was the only relevant structural rearrangement in the bone marrow cells of the patient.

Very few CN switches were observed either on chromosome 3 or the rest of the genome. Due to the poor quality of the DNA, massive whole genome sequencing was not performed. However, the genesis of this rearrangement was not due to chromothripsis, because we observed less than ten $\mathrm{CN}$ variations [6] along the entire chromosome.
The lack of mutation within TP53, previously reported as strongly associated with chromothripsis in AML [6], further reinforced this conclusion.

FISH analysis with chromosome paints for $3 p$ and $3 q$ revealed a complex reorganization of chromosome 3 (Figure 1b). Reiterative FISH assays with BAC (Roswell Park Cancer Institute [RPCI]-11 Human Male Bac Library, Buffalo, NY) and fosmid (WIBR2 Human Fosmid Library) probes, chosen according to the GRCh37/hg19 sequence and obtained from the BACPAC Resource Center (http:// bacpac.chori.org), allowed us to map four breakpoint regions.

The first region was mapped within the overlap between RP11-910P10, G248P80064E6 and G248P8385G8 (Figures 1c, d, g), upstream to the coding sequence of the FOXP1 gene (Figure 1g). FOXP1 encodes for a transcription factor playing important roles in the regulation of tissue-specific gene transcription during cell growth and differentiation [7].

The second breakpoint region was located within the overlapping clones RP11-90I13 (Figure 1c), RP11-722G11, and RP11-61G10, encompassing the $5^{\prime}$ portions of the non coding RNA LOC100302640 and LOC344595 genes, with opposite transcriptional orientation, and with unknown function (Figure 1g).

We conclude that an initial inversion event $[\operatorname{inv}(3)$ (p13q13.12)] led to the juxtaposition of the FOXP1 coding region to the 5 ' portion of a non coding RNA gene.

The third and forth breakpoints were mapped, respectively, within the overlapping region between RP11-1148C5, RP11-1105 J22, and RP11-626C4 (Figures 1e,g). They encompassed the TP63 gene (Figure 1g), and RP11-453G17 (Figure 1f,g), covering the first intron of the non-coding RNA BC031255 gene, with unknown function. TP63 is a member of the TP53 family of transcription factor genes, encoding by alternative promoters for two main isoforms, $\triangle N p 63(p 40)$ and TAp63. The TAp63 proteins transactivate the majority of the TP53 target promoters inducing cell cycle arrest and apoptosis. On the contrary, the $\Delta \mathrm{Np} 63$ isoforms seem to counteract the transactivation activities of TP53 and TAp63 proteins, possibly conferring a proliferative advantage on cancer cells [8]. Notably, the breakpoint within TP63 was mapped upstream to the $\triangle N p 63$ transcription start site (Figure 1g). 
The second inversion event $[\operatorname{inv}(3)(\mathrm{p} 12.2 \mathrm{q} 28)]$ led to the rearrangement of TP63 and $B C 031255$, with the same transcriptional orientation.

Interphase FISH with the RP11-910P10 clone also showed the splitting of the probe in $94 \%$ and $74 \%$ of cells, respectively in the BM and in the peripheral blood (PB) at diagnosis (data not shown). This result disclosed that the abnormal cell clone was already present in the $\mathrm{PB}$ at onset, confirming the aggressiveness of the MDS disease.

The lack of RNA material precluded the possibility of investigating genes expression patterns. However, we evaluated FOXP1 and TP63 expression by immunohistochemistry analysis (Figures 2 and 3 ). The patient was negative for the expression of all $\Delta \mathrm{N}$ isoforms of TP63 (with A00112 anti-p40 rabbit polyclonal antibody, Scytek, UT, USA) (data not shown). However, the patient was positive to the common region to all TP63 isoforms (with NCL-p63 anti-p63 mouse monoclonal antibody, Novocastra, Milan) (Figure 2a), as well as for FOXP1

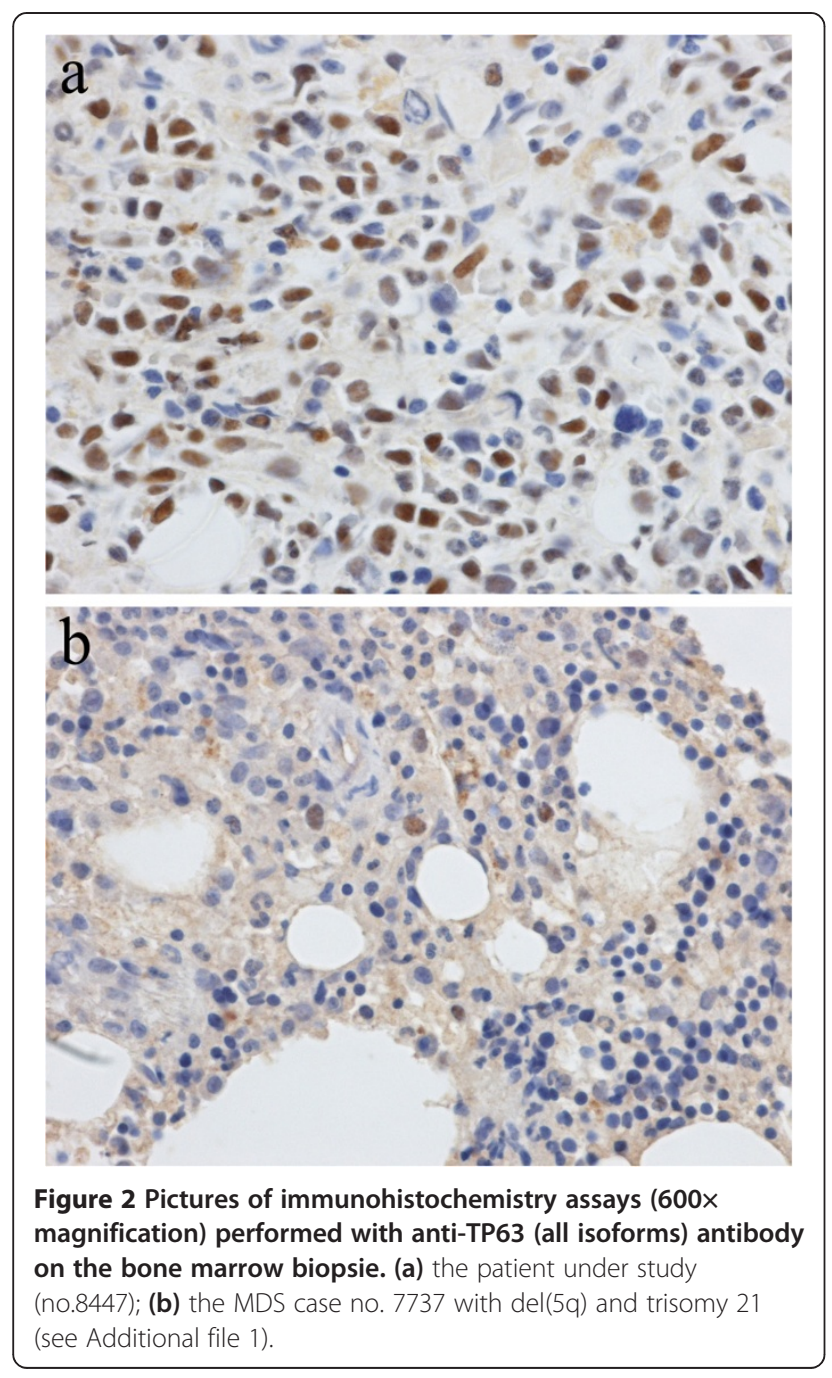

(with E19062 rabbit anti-FOXP1 polyclonal antibody, Spring Bioscience Corp., CA, USA) (Figure 3a). For FOXP1, we found a prevalent positivity of myeloid precursor cells, present in a high percentage in the bone marrow of the patient.

We performed the same analysis on four normal BM, as well as in additional MDS/AML cases with normal karyotypes, or with 5q- as a sole cytogenetic abnormality, or with additional changes (Additional file 1).

The overall results showed that TP63 was negatively expressed in all the control cases (Figure $2 \mathrm{~b}$ and Additional file 1). Conversely, FOXP1 was negatively expressed only in normal BM (Figure $3 \mathrm{~b}$ ) and one MDS case with a normal karyotype (Additional file 1). Of note, the remaining cases showed a variable level of protein expression (from 1 to 4 , Additional file 1), directly proportional to the percentage of myeloid precursor cells found in each case [level 1 (10\%); level 2 (10-50\%); level 3 (50-80\%); level 4 (more than 80\%). A low positivity level was occasionally found in megacaryoblasts, megacaryocytes, and in a few cases in rare erytroid precursors.

Our overall results showed that AML cases in our cohort displayed higher expression levels (from 2 to 4) of FOXP1 than MDS (from 0 to 3). In particular, our patient showed the highest FOXP1 expression level among MDS cases (level 3), i.e. she had a high percentage of positive myeloid precursors in her bone marrow.

Moreover, we observed that the majority of the MDS/ AML patients refractory to therapies, including our case, showed the highest FOXP1 expression levels [3-4 rather than $1-2$, respectively in $6 / 11$ (54\%) versus $3 / 9$ (33\%)]. According to these preliminary results, which need to be confirmed by additional research, we hypothesize a possible involvement of this gene in the progression of myeloid diseases.

\section{Conclusions}

To the best of our knowledge, we describe here the first MDS case with $5 \mathrm{q}$ - and a rearrangement of chromosome 3 involving FOXP1 and TP63.

FOXP1 was already described as fused to either the $P A X 5$ or the $A B L 1$ gene in B-ALL, and to the immunoglobulin heavy chain locus in lymphomas [7]. Strikingly, the breakpoint position in the present case was the same as in lymphomas accompanied by the gene upregulation. In myeloid malignancies, FOXP1 was reported as a target of deletion in both AML [9] and in myeloproliferative neoplasms [10].We also report here for the first time that FOXP1 is deregulated in MDS/AML cases. We presently have little information concerning the role of TP63 isoforms in myeloid cells. In B-cells, upregulation of TAp63 isoforms increased survival by activating BCL2 expression [11]. Mutations of TP63 were described only in CML blast crisis [12]. 


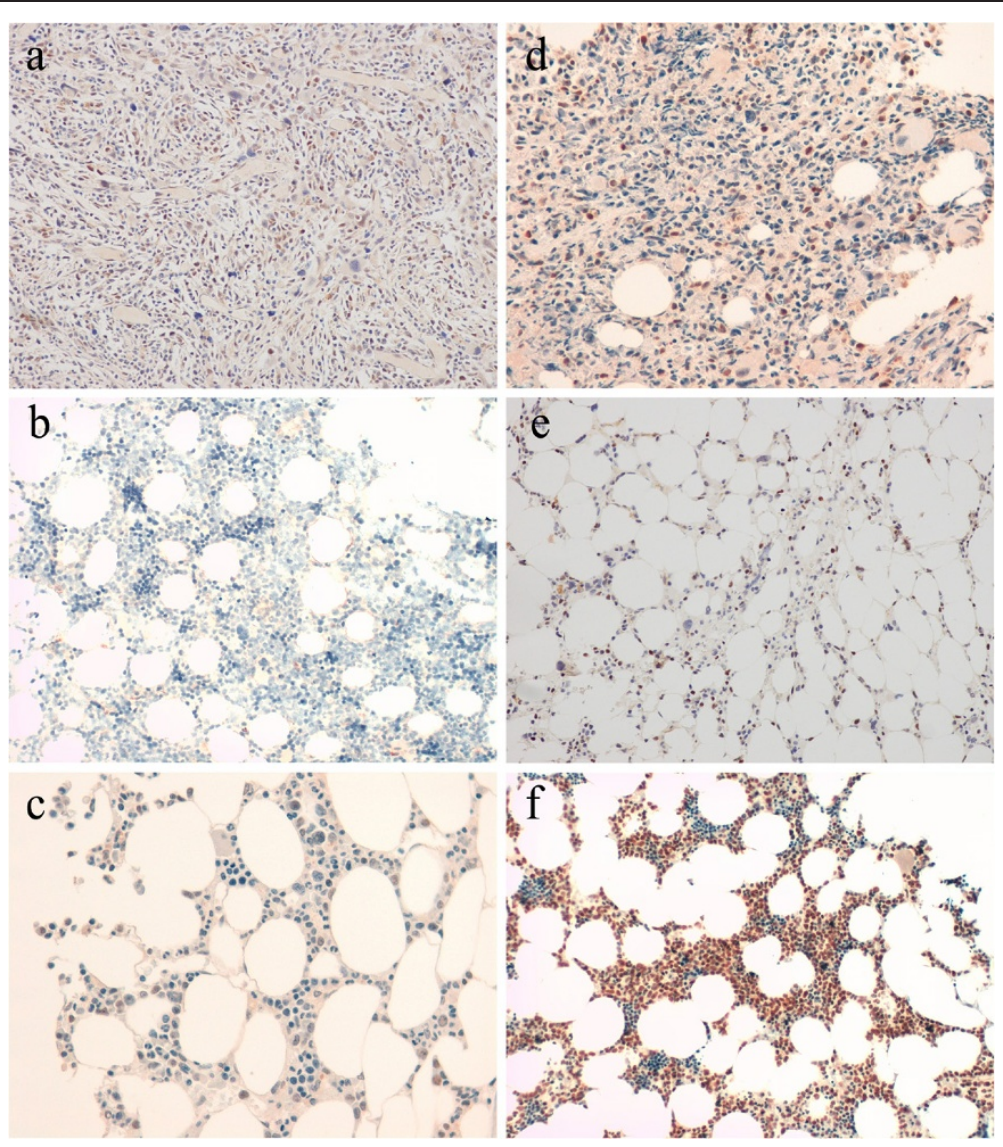

Figure 3 Pictures of immunohistochemistry assays (200x magnification) performed with anti-FOXP1 antibody showing different levels of protein expression in the bone marrow biopsies of investigated cases. (a) patient under study (level 3); (b) normal BM (level 0); (c) MDS case no. 558/10 with del(5q) as a sole cytogenetic abnormality (level 1); (d) MDS case no. 7737 with del(5q) and trisomy 21 (level 2); (e) MDS case no. 2374 with del(5q) and monosomy 7 (level 3); (f) AML case no. 635/12 with normal karyotype (level 4). (see Additional file 1).

In summary, we report here a notable MDS case, which rapidly evolved to AML, harboring $5 \mathrm{q}-$ and dysregulation of both FOXP1 and TP63.

We hypothesize that there is a possible role of FOXP1 and TP63 upregulation in the disease progression in this patient, as well as a potential involvement of FOXP1 in the evolution of MDS/AML and response to therapy, to be confirmed by additional experiments on a larger cohort of patients.

\section{Ethics statement}

This study was performed in agreement with the Declaration of Helsinki, and approved by the Ethical Committee at the National Cancer Research Centre "Giovanni Paolo II", Bari (Prot. No. 449/2013), and by the Ethical Committee at the "Seràgnoli" Institute of Hematology of Bologna (Prot. No. 253/2013/O).

Written informed consent was obtained from the patient for publication of this Case report and any accompanying images. A copy of the written consent is available for review by the Editor of this journal. Moreover, written informed consent was obtained for the control bone marrow samples and the additional MDS/AML cases.

\section{Additional file}

\section{Additional file 1: List of AML/MDS cases investigated for} immunohistochemistry analysis. The result for anti-FOXP1 antibody is reported in arabic numbers (from 0 to 4). Anti-TP40 and anti-TP63 antibodies result is expressed by + (positive) or - (negative).

\section{Abbreviations}

(MDS): Myelodysplastic Syndromes; (5q)]: Deletion 5q [del; (BM): Bone marrow (WBC): White blood cells; (Hb): Hemoglobin; (AML): Acute myeloid leukemia; (CN): Copy number; (FISH): Fluorescence in situ hybridization; (BAC): Bacterial Artificial Chromosome; (FOXP1): forkhead box P1; (TP63): Tumor protein p63; (PB): Peripheral blood; (JAK2): Janus kinase 2; (KMT2A): Lysine (K)-specific methyltransferase 2.

\section{Competing interests}

The authors declare that they have no competing interests.

\section{Authors' contributions}

ALA performed FISH analysis, and analysed the data; CLC provided the sample material and conceived the study; EM and CA evaluated immunohistochemistry results; PI and AG critically revised the manuscript; 
$\mathrm{CM}, \mathrm{NT}, \mathrm{GDT}$, and CM provided a substancial contribution to the acquisition of data for the study; CD performed immunohistochemistry analysis; $M C$ performed TP53 mutation analysis; LAM reviewed the TP53 mutation data; SS performed SNP array CGH analysis; ET analysed SNP array CGH data and edited the manuscript; CTS reviewed all the data, and wrote the manuscript. All authors read and approved the final manuscript.

\section{Acknowledgements}

This work was supported by the AIRC (Associazione Italiana per la Ricerca sul Cancro), and the MIUR (Ministero dell'Istruzione, dell'Università e della Ricerca). We thank Bruna De Benedet for technical assistance, and Prof. Roscoe Stanyon for English language editing.

\section{Author details}

'Department of Biology, University of Bari, Via G.Amendola 165/A, Bari 70126, Italy. ${ }^{2}$ UO Anatomia Patologica, Ospedale S. Martino, Belluno, Italy. ${ }^{3}$ Hematology Unit, University of Perugia, Polo Unico S.M. Misericordia, Perugia, Italy. ${ }^{4}$ Istituto Scientifico San Raffaele, Milan, Italy. ${ }^{5}$ Laboratory of Oncology, IRCCS "Casa Sollievo della Sofferenza", San Giovanni Rotondo, Italy. ${ }^{6}$ Center for Genome Research, Department of Biomedical Sciences, University of Modena and Reggio Emilia, Modena, Italy. ${ }^{7}$ Haematology Unit, Department of Medical and Experimental Oncology, IRCCS National Cancer

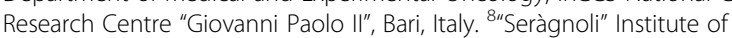
Hematology, Bologna University School of Medicine, Bologna, Italy.

Received: 5 November 2013 Accepted: 20 May 2014

Published: 3 June 2014

\section{References}

1. Kulasekararaj AG, Smith AE, Mian SA, Mohamedali AM, Krishnamurthy P, Lea NC, Gaken J, Pennaneach C, Ireland R, Czepulkowski B, Pomplun S, Marsh JC, Mufti GJ: TP53 mutations in myelodysplastic syndrome are strongly correlated with aberrations of chromosome 5 , and correlate with adverse prognosis. Br J Haematol 2013, 160(5):660-672.

2. Mallo M, Cervera J, Schanz J, Such E, Garcia-Manero G, Luno E, Steidl C, Espinet B, Vallespi T, Germing U, Blum S, Ohyashiki K, Grau J, Pfeilstöcker M, Hernández JM, Noesslinger T, Giagounidis A, Aul C, Calasanz MJ, Martín ML, Valent P, Collado R, Haferlach C, Fonatsch C, Lübbert M, Stauder R, Hildebrandt B, Krieger O, Pedro C, Arenillas L: Impact of adjunct cytogenetic abnormalities for prognostic stratification in patients with myelodysplastic syndrome and deletion 5q. Leukemia 2011, 25(1):110-120

3. Mallo M, Del Rey M, Ibanez M, Calasanz MJ, Arenillas L, Larrayoz MJ, Pedro C, Jerez A, Maciejewski J, Costa D, Nomdedeu M, Diez-Campelo M, Lumbreras E, González-Martínez T, Marugán I, Such E, Cervera J, Cigudosa JC, Alvarez S, Florensa L, Hernández JM, Solé F: Response to lenalidomide in myelodysplastic syndromes with del(5q): influence of cytogenetics and mutations. $\mathrm{Br} J$ Haematol 2013, 162(1):74-86.

4. Greenberg PL, Tuechler H, Schanz J, Sanz G, Garcia-Manero G, Sole F, Bennett JM, Bowen D, Fenaux P, Dreyfus F, Kantarjian H, Kuendgen A, Levis A, Malcovati L, Cazzola M, Cermak J, Fonatsch C, Le Beau MM, Slovak ML, Krieger O, Luebbert M, Maciejewski J, Magalhaes SM, Miyazaki Y, Pfeilstöcker M, Sekeres M, Sperr WR, Stauder R, Tauro S, Valent P: Revised international prognostic scoring system for myelodysplastic syndromes. Blood 2012, 120(12):2454-2465.

5. Storlazzi CT, Albano F, Dencic-Fekete M, Djordjevic V, Rocchi M: Late-appearing pseudocentric fission event during chronic myeloid leukemia progression. Cancer Genet Cytogenet 2007, 174(1):61-67.

6. Rausch T, Jones DT, Zapatka M, Stutz AM, Zichner T, Weischenfeldt J, Jager N, Remke M, Shih D, Northcott PA, Pfaff E, Tica J, Wang Q, Massimi L, Witt H, Bender S, Pleier S, Cin H, Hawkins C, Beck C, von Deimling A, Hans V, Brors B, Eils R, Scheurlen W, Blake J, Benes V, Kulozik AE, Witt O, Martin D: Genome sequencing of pediatric medulloblastoma links catastrophic DNA rearrangements with TP53 mutations. Cell 2012, 148(1-2):59-71.

7. Katoh M, Igarashi M, Fukuda H, Nakagama H: Cancer genetics and genomics of human FOX family genes. Cancer Lett 2013, 328(2):198-206.

8. Candi E, Dinsdale D, Rufini A, Salomoni P, Knight RA, Mueller M, Krammer PH, Melino G: TAp63 and DeltaNp63 in cancer and epidermal development. Cell Cycle 2007, 6(3):274-285.

9. Bullinger L, Kronke J, Gaidzik V, Dohner H, Dohner K: Comment on 'Integrative genomic profiling of human prostate cancer'. Leukemia 2010, 24(11):1970-1972.
10. Klampfl T, Harutyunyan A, Berg T, Gisslinger B, Schalling M, Bagienski K, Olcaydu D, Passamonti F, Rumi E, Pietra D, Jäger R, Pieri L, Guglielmelli P, lacobucci I, Martinelli G, Cazzola M, Vannucchi AM, Gisslinger H, Kralovics R: Genome integrity of myeloproliferative neoplasms in chronic phase and during disease progression. Blood 2011, 118(1):167-176.

11. Shachar I, Haran M: The secret second life of an innocent chaperone: the story of CD74 and B cell/chronic lymphocytic leukemia cell survival. Leuk Lymphoma 2011, 52(8):1446-1454.

12. Yamaguchi $H$, Inokuchi $K$, Sakuma Y, Dan K: Mutation of the $p 51 / p 63$ gene is associated with blastic crisis in chronic myelogenous leukemia. Leukemia 2001, 15(11):1729-1734.

doi:10.1186/1471-2407-14-396

Cite this article as: L'Abbate et al.: FOXP1 and TP63 involvement in the progression of myelodysplastic syndrome with $5 q$ - and additional cytogenetic abnormalities. BMC Cancer 2014 14:396.

\section{Submit your next manuscript to BioMed Central and take full advantage of:}

- Convenient online submission

- Thorough peer review

- No space constraints or color figure charges

- Immediate publication on acceptance

- Inclusion in PubMed, CAS, Scopus and Google Scholar

- Research which is freely available for redistribution

Submit your manuscript at www.biomedcentral.com/submit
C Biomed Central 